\title{
Avaliação da Distribuição Espacial das Estações de Observação Climática de Superfície de Santa Catarina Ativas em 2020, Segundo As Orientações da Organização Mundial de Meteorologia (OMM)
}

\author{
Luiz Fernando de Novaes Vianna $^{1}$ (D), Cristina Pandolfo $^{1}$ (D), Everton Blainski ${ }^{1}$ (D), \\ Hamilton Justino Vieira ${ }^{1}$ \\ ${ }^{1}$ Centro de Informações Ambientais e de Hidrometeorologia, Empresa de Pesquisa Agropecuária \\ e Extensão Rural de Santa Catarina, Florianópolis, SC, Brasil.
}

Recebido em: 15 de Fevereiro de 2021 - Aceito em: 30 de Junho de 2021

\begin{abstract}
Resumo
A Organização Mundial de Meteorologia (OMM) orienta que cada estação de observação climática de uma rede de estações de superfície represente "o estado da atmosfera de uma vasta região". A dimensão dessa região, ou área de representatividade, pode variar de $2.000 \mathrm{~km}^{2}$ a $10.000 \mathrm{~km}^{2}$, dependendo da heterogeneidade do relevo. Regiões com relevo homogêneo podem ter uma menor densidade de estações (uma estação a cada $10.000 \mathrm{~km}^{2}$ ), enquanto regiões com relevo acidentado e com muita variação de altitude precisam de uma densidade maior de estações (uma estação a cada $2.000 \mathrm{~km}^{2}$ ). O objetivo desse trabalho foi comparar a heterogeneidade do relevo de Santa Catarina com a distribuição das 187 estações de observação climática ativas em 01/08/2020, analisar se as densidades são representativas e propor uma distribuição que represente a heterogeneidade do relevo de Santa Catarina, segundo as orientações da OMM. Três métricas de terreno (altitude, desvio padrão da altitude e índice de posição topográfica) foram utilizadas para mapear a heterogeneidade do relevo. O mapeamento foi feito no ArcGis Pro (v. 2.1.2) utilizando-se análise de componentes principais e análise de agrupamento. Os resultados apontam que, em termos quantitativos, o número atual de estações de observação climática pode atender às orientações da OMM, porém é necessário que se faça uma distribuição espacial mais adequada, para que a rede cubra a maior variabilidade fisiográfica possível.
\end{abstract}

Palavras-chave: meteorologia, monitoramento, previsão do tempo, climatologia.

\section{Evaluation of the Spatial Distribution of Active Surface Climatic Observation Stations in Santa Catarina (2020) According to the Guidelines of the World Meteorological Organization (WMO)}

\begin{abstract}
The World Meteorological Organization (WMO) advises that each surface climatic observation station in a network represents "the state of the atmosphere of a vast region". The extension of this region, or representative area, can vary from $2,000 \mathrm{~km}^{2}$ to $10,000 \mathrm{~km}^{2}$, depending on the heterogeneity of the relief. Regions with homogeneous relief may have a lower density of stations (one station every $10,000 \mathrm{~km} 2$ ), while regions with rugged relief needs a higher density of stations (one station every 2,000 km2). The objective of this work was to compare the heterogeneity of Santa Catarina relief with the current (2020/08/01) distribution of 187 active surface climatic observation stations, and to suggest a network stations that represent the heterogeneity of relief according to the WMO guidelines. Three terrain metrics (altitude, standard deviation of altitude and topographic position index) were used to map the heterogeneity of the relief. The mapping was done in ArcGis Pro (v. 2.1.2) using principal component analysis and cluster analysis. The results show that, in quantity, the current number of meteorological stations can meet the WMO guidelines, however, it is necessary to make a more adequate spatial distribution to cover the maximum heterogeneity of the relief.
\end{abstract}

Keywords: metheorology, monitoring, wheather, climate changes.

Autor de correspondência: Luiz Fernando de Novaes Vianna, vianna@epagri.sc.gov.br. 


\section{Introdução}

Segundo a Organização Mundial de Meteorologia OMM (WMO, 2007), a principal questão que envolve a escolha de um local para instalação de uma estação de observação climática é: "Is the site representative of the required meteorological data?", ou seja, qual a área de abrangência representada pelos dados coletados naquele local? Essa resposta é complexa e envolve diversos parâmetros físicos, relacionados principalmente à heterogeneidade da paisagem de entorno do local (ex. relevo, vegetação e urbanização). Além disso, a escolha do local também depende de aspectos logísticos e de infraestrutura (acesso viário, energia e comunicação) (Blainski et al., 2012).

A OMM define como estações de observação climáticas, uma série de tipos de estações (ex. estações sinóticas, estações climatológicas e estações agrícolas) que coletam dados climáticos segundo objetivos diversos (WMO, 2014). O estado de Santa Catarina classifica as estações de observação climáticas em estações meteorológicas e estações agrometeorológicas (Blainski et al., 2012; Braga et al., 2015). O que as diferencia são a quantidade de variáveis medidas em cada uma, dependendo do objetivo. Segundo os autores, tanto as estações meteorológicas quanto as estações agrometeorológicas são implantadas e mantidas seguindo os padrões de qualidade definidos pela OMM.

Não há normas nem métodos definidos para a escolha do local nem para calcular a quantidade de estações representativas de uma região, mas a OMM orienta que cada estação de observação sinótica deve estar localizada em um ponto que represente "o estado da atmosfera de uma vasta região" (WMO, 2007). Segundo a OMM, a dimensão dessa região, ou área de representatividade, pode variar de $2.000 \mathrm{~km}^{2}$ a $10.000 \mathrm{~km}^{2}$, dependendo da heterogeneidade do relevo. Regiões com relevo homogêneo podem ter uma menor densidade de estações (uma estação a cada $10.000 \mathrm{~km}^{2}$ ), enquanto regiões com relevo acidentado e com muita variação de altitude precisam de uma densidade maior de estações (uma estação a cada $2.000 \mathrm{~km}^{2}$ ).

O Estado de Santa Catarina possui uma rede de estações de observação climática que atende a diversas instituições e setores da sociedade. Dentre os principais objetivos da rede está o monitoramento e a previsão climática, com foco na agricultura e na previsão de desastres naturais (Blainski et al., 2012).

De a cordo com a classificação de Köppen, o estado de Santa Catarina apresenta clima mesotérmico úmido, sem estação seca (Cf), que pode ser subdividido em Cfa e $\mathrm{Cfb}$, diferenciados por apresentarem verão quente e verão fresco, respectivamente. Em escala estadual, Braga e Ghellere (1999) propuseram uma divisão em 5 zonas e 10 sub-zonas climáticas, considerando a climatologia de 40 estações de observação climática, a hipsometria e o mapeamento fitogeográfico do estado. Mesmo analisando a altitude, o método implementado pelos autores não foi suficiente para diferenciar nuances do relevo em escala local, resultando em uma proposta de divisão do estado de Santa Catarina em 11 regiões climáticas.

Como o relevo de Santa Catarina é heterogêneo (Santa Catarina, 2014), é importante que a distribuição espacial das estações de observação climática represente a maior variabilidade possível de feições fisiográficas e, consequentemente, as diversas nuances climáticas associadas.

O objetivo principal desse trabalho foi analisar a distribuição espacial das estações de observação climática ativas em agosto de $2020 \mathrm{em}$ Santa Catarina segundo as recomendações da OMM. Para isso alguns objetivos específicos foram considerados: (i) avaliar a quantidade e a densidade das estações no território do estado; (ii) comparar a atual distribuição espacial das estações de observação climática ativas com a heterogeneidade do relevo de Santa Catarina; (iii) avaliar a viabilidade logística dos sítios de instalação de futuras estações considerando o alcance de sinal de telefonia móvel e o acesso viário; e (iv) propor uma rede que represente a heterogeneidade do relevo de acordo com as recomendações mínimas da OMM e a disponibilidade atual de estações em Santa Catarina.

\section{Materiais e Métodos}

O trabalho foi realizado em três etapas: (i) Análise da densidade das estações de observação climática no território do estado de Santa Catarina; (ii) estimativa do número de estações de observação climática por região e sub-regiões fisiográficas homogêneas; (iii) proposta de distribuição espacial das estações de observação climática de acordo com as especificações da OMM e a capacidade logística de sinal de telefonia móvel e acesso viário (Fig. 1).

Na primeira etapa foi feita uma análise de densidade das estações de observação climática considerando o território do estado de Santa Catarina e as classes das estações (Blainski et al., 2012; Braga et al., 2015). Os pontos das estações de observação climática ativas em 01/08/2020 foram fornecidos pelo Centro de Informações Ambientais e de Hidrometeorologia (Ciram) da Empresa de Pesquisa Agropecuária e Extensão Rural de Santa Catarina (Epagri) em uma planilha contendo o código da estação, as coordenadas geográficas e a classificação das estações. Os pontos das estações foram utilizados para gerar um mapa de densidade através da ferramenta de cálculo de densidade de pontos do ArcGis Pro (v. 2.1.2) (ESRI, 2020). A densidade foi calculada tanto para o conjunto de estações como para as duas classes de estações separadamente (meteorológicas e agrometeorológicas), considerando um raio de al- 


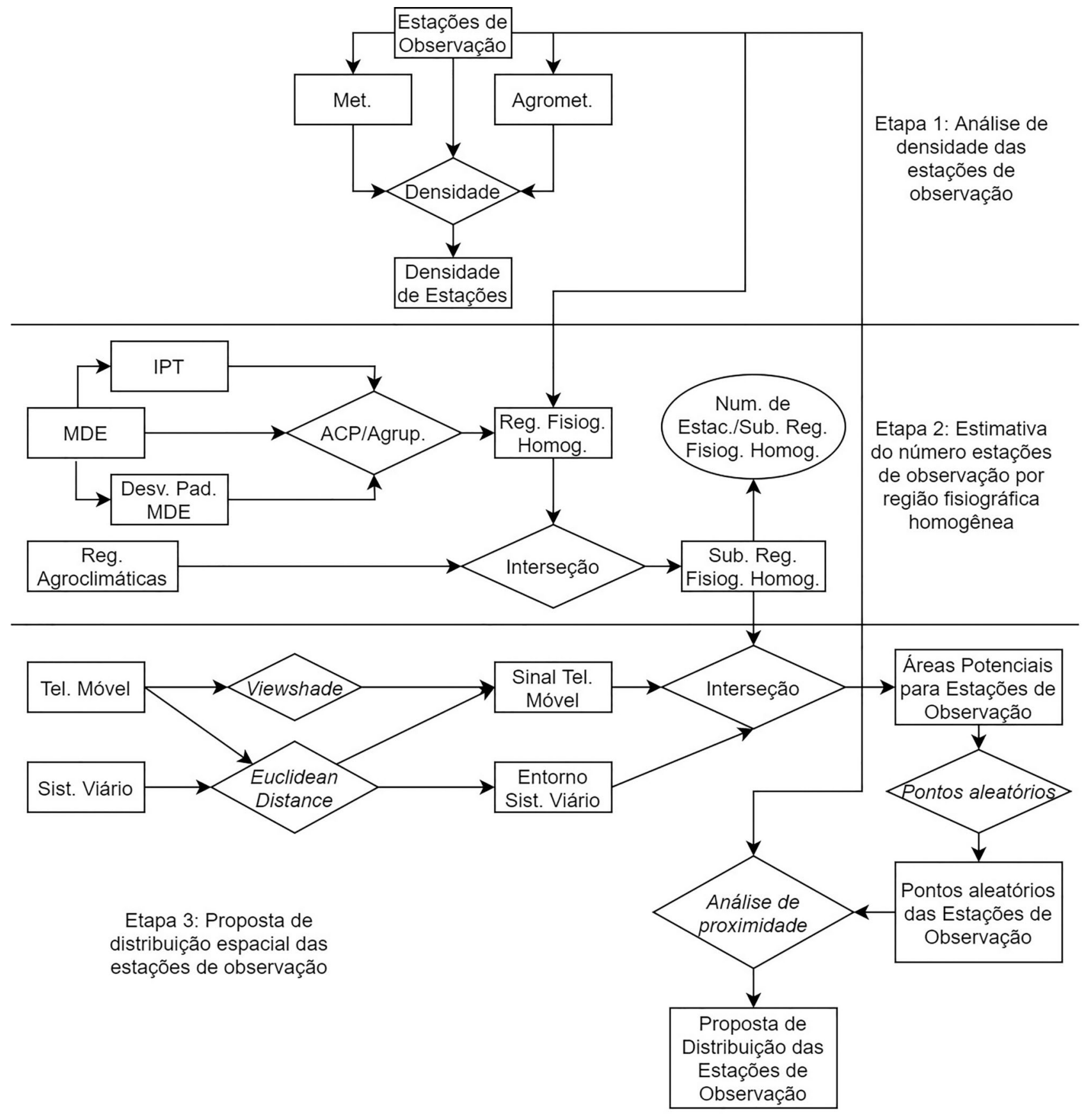

Figura 1 - Fluxograma das três etapas do método.

cance de $25 \mathrm{~km}$, que corresponde à área de $2.000 \mathrm{~km}^{2}$ sugerida pela OMM.

$\mathrm{Na}$ segunda etapa foi feita uma análise da heterogeneidade do relevo do estado de Santa Catarina e uma estimativa do número de estações necessárias para representar essa heterogeneidade considerando a área mínima de $2.000 \mathrm{~km}^{2}$, sugerida pela OMM. A heterogeneidade do relevo foi analisada através do mapeamento das regiões fisiográficas homogêneas, considerando três métricas de terreno: altitude, desvio padrão da altitude e índice de posição topográfica (Amatulli et al., 2018). Para o mapeamento das regiões fisiográficas homogêneas foi utilizado o modelo digital de elevação (MDE) do Aster, com pixel de 30 metros (NASA/METI/AIST/ASTER Science Team, 2001). Além da altitude, o MDE foi utilizado para gerar as métricas de desvio padrão da altitude e índice de posição topográfica, segundo o método proposto por Jenness et al. (2007) e aplicado por Tagil e Jenness (2008). 
O mapa das regiões fisiográficas homogêneas foi feito aplicando-se as técnicas de análise multivariada de componentes principais e agrupamento do ArcGis Pro (v. 2.1.2) (ESRI, 2020a; ESRI, 2020b). O resultado da análise de componentes principais foi utilizado para gerar as classes fisiográficas através da técnica de classificação não supervisionada de agrupamento. O número de classes foi definido estatisticamente segundo o método proposto por Kassambra (2017).

O mapa das regiões fisiográficas homogêneas foi cruzado com mapa das sub-zonas climáticas de Santa Catarina (Braga e Ghellre, 1999) e as classes fisiográficas coincidentes com cada região agroclimática receberam seus respectivos códigos. O cruzamento gerou as subregiões fisiográficas que foram nomeadas de acordo com as siglas das sub-zonas climáticas (Braga e Ghellre, 1999).

As estações de observação climática foram cruzadas com o mapa das sub-regiões fisiográficas homogêneas e foi calculada a densidade de estações para cada região e sub-região.

De acordo com a área de cada região e de cada subregião fisiográfica, foi feito um cálculo do número de estações necessário para atender à densidade mínima de uma estação para cada $2000 \mathrm{~km}^{2}$. Quando a área da região ou da sub-região foi menor que $2000 \mathrm{~km}^{2}$, considerou-se a necessidade de uma estação para representá-la. A diferença entre o número mínimo de estações necessárias e a quantidade de estações existentes representa a quantidade de estações em déficit ou em excesso, em cada região ou sub-região fisiográfica.

$\mathrm{Na}$ terceira etapa foi feita uma proposta de distribuição espacial para as estações ativas em Santa Catarina. Para identificar os locais ideais para disposição das estações e propor uma rede que represente a variabilidade fisiográfica utilizando as estações existentes, foram considerados a heterogeneidade do relevo, representada pelas sub-regiões fisiográficas, o alcance do sinal da telefonia móvel e a condição de acesso através da malha viária.

A malha de pontos de localização das torres de telefonia móvel foi obtida na página da Associação Brasileira de Telecomunicações. A partir da localização das torres foi feito o mapa de alcance de sinal, com base no campo de visada de cada torre sobre o relevo em um raio de alcance de $35 \mathrm{~km}$, que é a distância média do alcance do sinal em linha reta, dependendo da potência de transmissão. O mapa foi gerado no ArcGis Pro (v. 2.1.2) através das ferramentas Viweshade e Euclidean distance (ESRI, 2020c, ESRI, 2020d).

A malha viária utilizada foi obtida na página do Geofrabrik. A proximidade máxima considerada ideal para o acesso às estações no entorno da malha viária foi de $300 \mathrm{~m}$ a partir do eixo de cada via. Essa área foi calculada utilizando a ferramenta de Euclidean distance do ArcGis Pro (v. 2.1.2) (ESRI, 2020d).
O mapa das áreas potenciais para implantação das estações foi gerado através do cruzamento (interseção) entre o mapa das sub-regiões fisiográficas, o mapa de alcance de sinal da telefonia móvel e o mapa da área de entorno da malha viária. A partir do mapa das áreas potenciais foram gerados 187 pontos aleatórios condicionados a representarem $100 \%$ das sub-regiões fisiográficas. Os pontos aleatórios foram gerados através da ferramenta Create random points do ArGis Pro (v. 2.1.2) (ESRI, 2020e), condicionando haver, no mínimo, um ponto para cada sub-região fisiográfica. No caso de sub-regiões com área superior a $2.000 \mathrm{~km}^{2}$, os pontos no seu interior foram gerados de forma a se situarem a pelo menos $25 \mathrm{~km}$ de distância entre si.

Após a geração automática dos pontos aleatórios, foi feita uma análise de proximidade com as estações ativas existentes. Os pontos localizados a menos de $25 \mathrm{~km}$ de distância de uma estação ativa já existente, dentro da mesma sub-região fisiográfica, foram deslocados até o ponto da estação pré-existente, evitando-se assim a necessidade de troca de local da estação.

Os pontos que não estão localizados na mesma subregião fisiográfica da estação mais próxima, tiveram suas coordenadas associadas às coordenadas e à distância das estações mais próximas num raio de $25 \mathrm{~km}$, para auxiliar na estratégia de realocação.

\section{Resultados e discussão}

O estado de Santa Catarina possuía, em agosto de 2020, 187 estações de observação climática ativas, sendo 71 meteorológicas e 116 agrometeorológicas (Fig. 2). O mapa de densidade representa os locais cobertos por uma ou mais estações em uma área de $2000 \mathrm{~km}^{2}$, correspondente a um raio aproximado de $25 \mathrm{~km}$. A maior parte do estado está representada por, pelo menos, uma estação a cada $2.000 \mathrm{~km}^{2}$ (áreas em tom de azul), mas ainda existem áreas desprovidas de cobertura, enquanto outras estão cobertas por até 10 estações (áreas em vermelho).

Avaliando-se a densidade de estações meteorológicas (Fig. 2B), a área desprovida de cobertura é maior, porém a distribuição espacial das estações é mais homogênea por toda a área do território do Estado. Em relação à densidade de estações agrometeorológicas (Fig. 2C) observam-se sete regiões com densidade superior a 3 estações por $2000 \mathrm{~km}^{2}$ e quatro regiões com densidade superior a 5 estações por $2000 \mathrm{~km}^{2}$. A concentração de estações agrometeorológicas nas áreas de maior densidade se justifica pela necessidade de coleta de dados meteorológicos para projetos de pesquisa agronômica e monitoramento de culturas específicas (Blainski et al., 2012).

Segundo os documentos técnicos da Epagri (Blainski et al., 2012; Braga et al., 2015) os dados climáticos coletados tanto através das estações meteorológicas quanto através das estações agrometeorológicas 

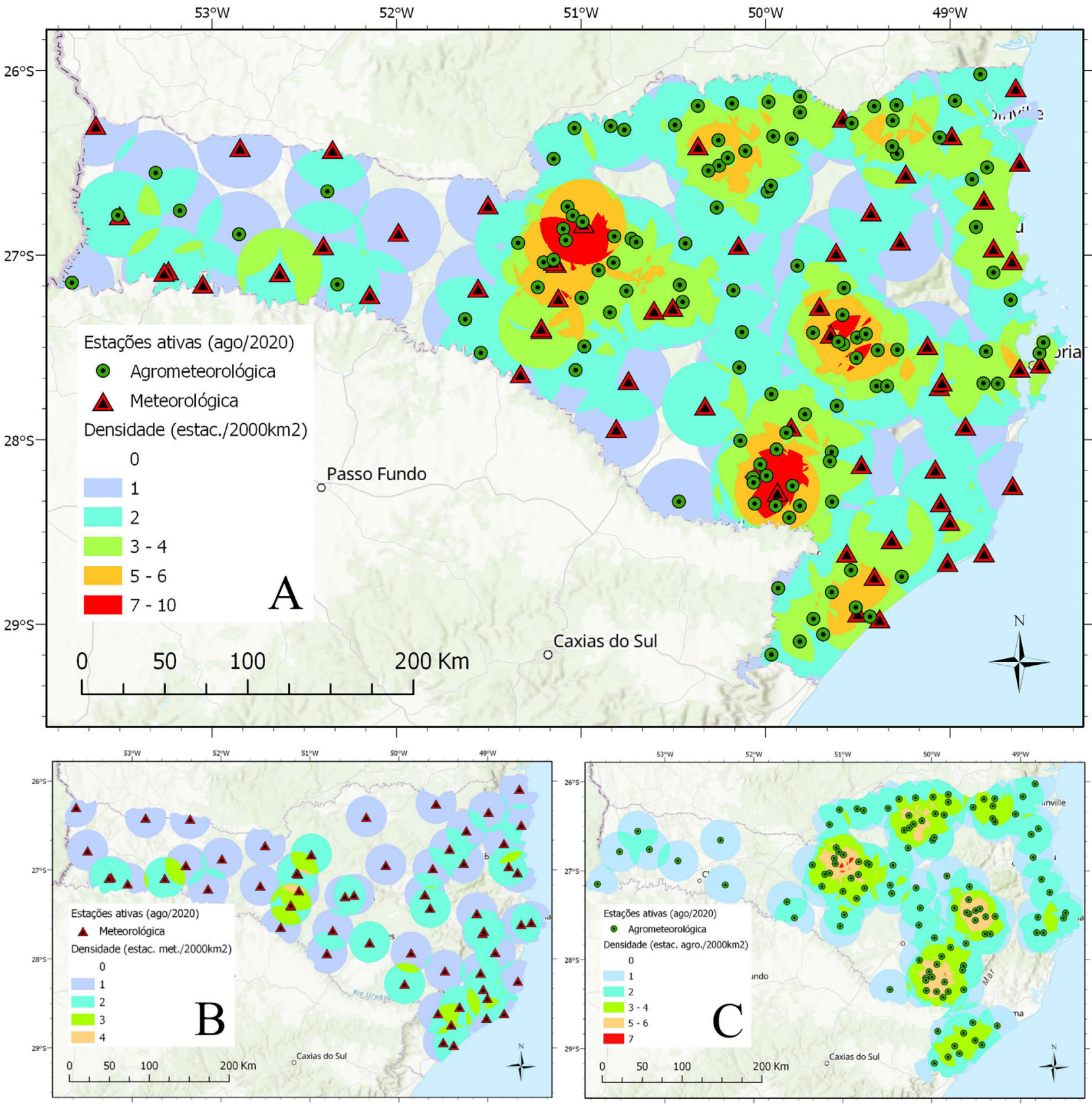

Figura 2 - Mapa da distribuição espacial e da densidade das estações de observação climática ativas em agosto de 2020 em Santa Catarina: a) densidade considerando todas as estações; b) densidade de estações meteorológicas; c) densidade de estações agrometeorológicas. As cores representam o número de estações que cobrem cada área em um raio de aproximadamente $25 \mathrm{~km}\left(2.000 \mathrm{~km}^{2}\right)$.

possuem a mesma qualidade, garantida pela fidelidade aos padrões de instalação e manutenção da OMM. Porém, como as estações agrometeorológicas possuem objetivos distintos, como, por exemplo, atender a projetos de pesquisa, alguns equipamentos extras podem ser instalados fora dos padrões da OMM (Blainski et al., 2012), sem que seus dados estejam disponíveis para uso geral. Como as variáveis climáticas tradicionais (precipitação, pressão atmosférica, radiação solar, temperatura do ar, umidade relativa e vento) são coletadas seguindo os padrões da OMM em ambas as classes de estações, as análises que seguem foram feitas considerando tanto as estações meteorológicas quanto as estações agrometeorológicas componentes de uma mesma rede de estações de observação climática.

Por ser um estado com relevo heterogêneo (Santa Catarina, 2014), a distribuição espacial das estações não deve ser regular, ou seja, em áreas com maior variação de altitude a densidade de estações deve ser maior que nas áreas onde o relevo é menos acidentado. Mas não é isso 
que se observa. Há uma maior concentração de estações nas cotas altimétricas abaixo de $200 \mathrm{~m}$ e nas cotas situadas entre 800 e 1000 m (Fig. 3A). Essas são as áreas que compõem a planície litorânea e o planalto de Santa Catarina, com de relevo que apresenta índices topográficos de terreno entre -137 (Vales), 0 (Planícies/Planaltos) e 45 (encostas inferiores) (Fig. 3C) e pouca variação de altitude (Fig. 3D, desvio padrão da altitude entre $-0,22$ e 0,22 ). Essas condições de relevo apresentam maior homo- geneidade, logo necessitariam de uma densidade menor de estações. Por outro lado, essas áreas concentram as estações agrometeorológicas, que segundo Blainski et al., (2012) estão associadas a projetos de pesquisa e monitoramento climático para alertas fitossanitários de culturas importantes para o Estado, como a maçã, a banana, o tomate e a cebola. Por isso é necessário mapear as diversas regiões e sub-regiões fisiográficas e avaliar a distribuição espacial das estações sobre cada uma delas. É preciso ava-
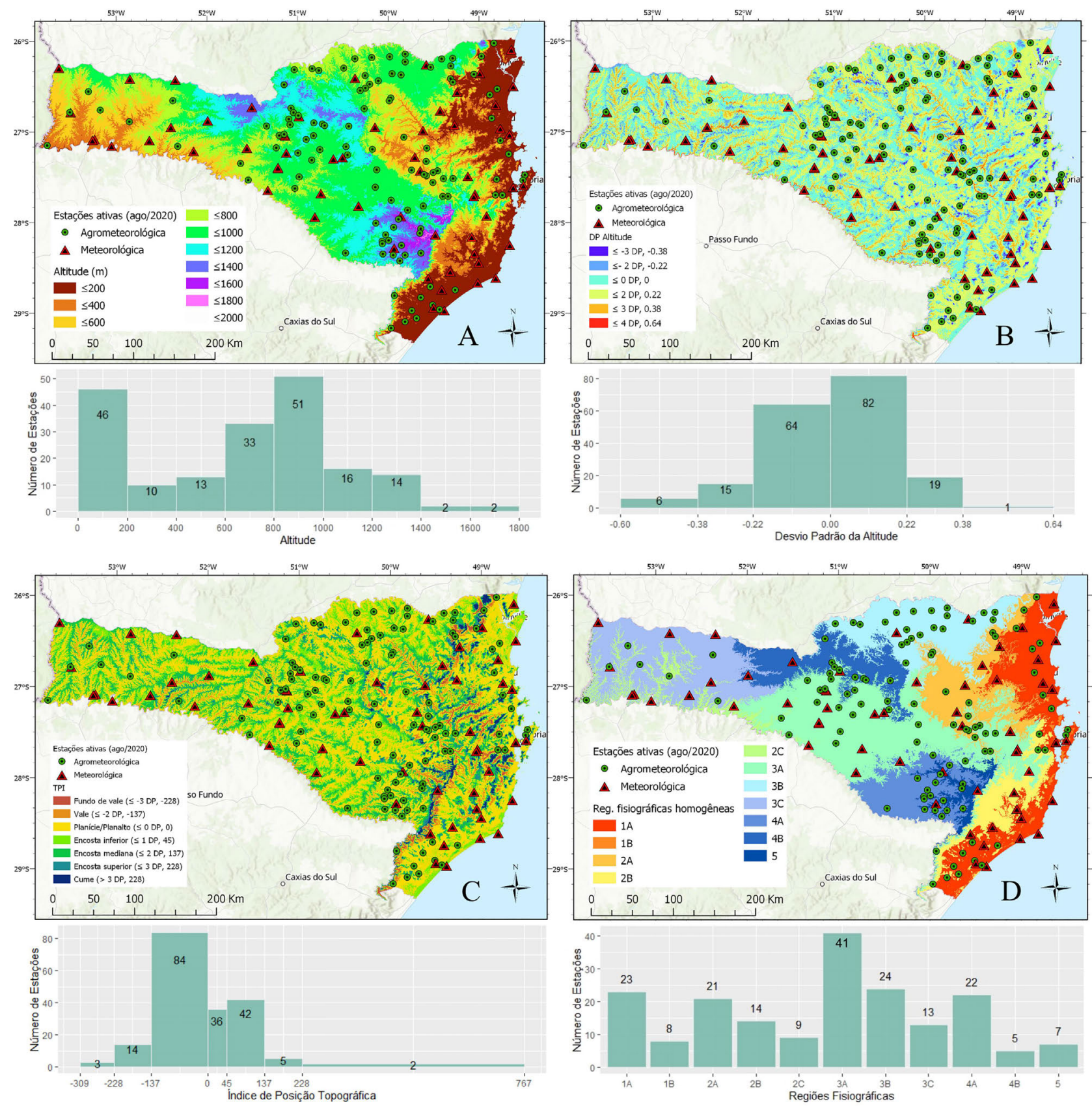

Figura 3 - Distribuição espacial das estações de observação climática ativas em agosto de 2020 em Santa Catarina sobre os mapas de altimetria (A), desvio padrão da altimetria (B), índice de posição topográfica (TPI) (C) e sobre o mapa das regiões fisiográficas homogêneas (D). Os histogramas indicam o número de estações por classe de cada mapa. 
liar também os objetivos de cada estação em relação ao sítio onde foram instaladas.

Em escala estadual, as 11 regiões fisiográficas estão quantitativamente atendidas pelo número mínimo de uma estação para cada $2.000 \mathrm{~km}^{2}$, sugerido pela OMM (Tabela 1, Fig. 3D). A região 4B é a que apresenta a menor diferença entre a quantidade de estações existentes e a quantidade de estações necessárias, com apenas uma estação excedente. Já a região $3 \mathrm{~A}$ apresenta um excedente de 29 estações.

O excedente quantitativo de estações em nível estadual não garante uma representação espacial integral em nível regional, visto que a fisiografia de cada região varia. Enquanto as regiões 1A, 3A e 3B, que possuem relevo mais homogêneo, concentram $45 \%$ das estações, as demais regiões, que são cortadas por vales e montanhas que variam de altitude bruscamente em poucos quilômetros de distância, estão sub representadas. Essas diferenças podem ser observadas nas sub-regiões fisiográficas apresentadas na Fig. 4, que são responsáveis por nuances microclimáticas que só podem ser identificadas com uma densidade maior e uma distribuição espacial das estações de observação climática condizente com a heterogeneidade fisiográfica.

O número mínimo que o estado de Santa Catarina necessita para cobrir $100 \%$ do território com estações de observação climática que representem uma área mínima de $2000 \mathrm{~km}^{2}$ de cada uma das 98 sub-regiões fisiográficas é de 114 estações. Em agosto de 2020 a rede de estações de observação climática de Santa Catarina contava com 187 estações, uma quantidade suficiente para prover o estado com dados meteorológicos padronizados internacionalmente. Porém existem áreas com um número excessivo de estações e áreas sem cobertura, o que torna necessário

Tabela 1 - Quantidade de estações de observação climática existentes em agosto de 2010, quantidade de estações de observação climática necessárias e diferença entre a quantidade de estações de observação climática necessárias e a quantidade de estações de observação climática existentes por classe fisiográfica em Santa Catarina.

\begin{tabular}{lcccc}
\hline $\begin{array}{l}\text { Classe fisio- } \\
\text { gráfica }\end{array}$ & $\begin{array}{c}\text { Área } \\
\left(\mathrm{km}^{2}\right)\end{array}$ & $\begin{array}{c}\text { Estações exis- } \\
\text { tentes - Ee }(08 / \\
2020)\end{array}$ & $\begin{array}{c}\text { Estações } \\
\text { necessárias - } \\
\text { En }\end{array}$ & $\begin{array}{c}\text { Diferença } \\
(\text { Ee - En) }\end{array}$ \\
\hline 1A & 13360 & 23 & 7 & 16 \\
1B & 311 & 8 & 1 & 7 \\
2A & 8763 & 21 & 4 & 17 \\
2B & 5028 & 14 & 3 & 11 \\
2C & 3541 & 9 & 2 & 7 \\
3A & 23412 & 41 & 12 & 29 \\
3B & 10973 & 24 & 5 & 19 \\
3C & 13288 & 13 & 7 & 6 \\
4A & 6824 & 22 & 3 & 19 \\
4B & 7558 & 5 & 4 & 1 \\
5 & 2046 & 7 & 1 & 6 \\
\hline
\end{tabular}

um ajuste espacial na distribuição das estações para atender às normas da OMM. Na Fig. 5, observa-se a necessidade de equilibrar o excedente de estações das áreas verdes com o déficit nas áreas em amarelo, laranja e vermelho.

Na Tabela 2 estão os cálculos necessários para quantificar o número de estações necessárias em cada subregião. As sub-regiões com diferença positiva, apresentam excesso de estações. Por outro lado, aquelas com diferença negativa necessitam daquele número mínimo de estações.

A proposta de realocação das estações com base na heterogeneidade do relevo depende de 188 estações (Fig. 6), uma a mais do que se tem hoje em operação. Para ajustar a rede de estações de observação climática do Estado de Santa Catarina de forma a representar a variabilidade de relevo, com o número atual de estações ativas (187), é necessário redistribuir algumas estações das áreas com alta densidade para as áreas mais desprovidas e acrescentar mais uma estação à rede. Mas o ajuste não é simples, uma vez que envolve decidir entre estações que devem ser mantidas e aquelas que podem ser remobilizadas.

Um dos aspectos mais relevantes para definir que estações podem ser trocadas de sítio é a análise da série histórica (Vianna et al., 2018). Estações com séries históricas significativas devem ser mantidas em seus sítios originais. Mas é preciso definir critérios estatísticos e científicos para auxiliar na avaliação a respeito da significância das séries históricas. Qual é o período mínimo para se considerar uma estação passível ou não de mudança de sítio? Segundo Vianna et al. (2018), as estações automáticas atualmente ativas em Santa Catarina possuem séries históricas que variam de poucos meses a até 22 anos, com uma média de 5,6 anos de dados.

Outro aspecto importante a ser considerado é o potencial logístico mínimo para a instalação e a manutenção das estações de observação climática. Segundo Blainski et al. (2012) o acesso viário e a existência de sinal de telefonia móvel são fatores indispensáveis para selecionar um sítio para instalação de uma estação de observação climática. Na Fig. 6 é apresentada uma proposta de realocação espacial das estações de observação climática de modo que todas as sub-regiões fisiográficas estejam representadas por pelo menos uma estação a cada $2.000 \mathrm{~km}^{2}$ (Fig. 6A), em áreas com acesso viário e cobertura de sinal de telefonia móvel (Fig. 6B).

Há 42 estações atualmente ativas, coincidentes espacialmente com as estações propostas. Há 15 estações atualmente ativas localizadas entre 100 e $500 \mathrm{~m}$ das estações propostas, porém em sub-regiões fisiográficas distintas. As 130 estações ativas restantes estão a mais de $500 \mathrm{~m}$ das estações propostas e em sub-regiões fisiográficas distintas.

Para executar a realocação das estações ativas é preciso considerar a distância, a sub-região fisiográfica a ser 


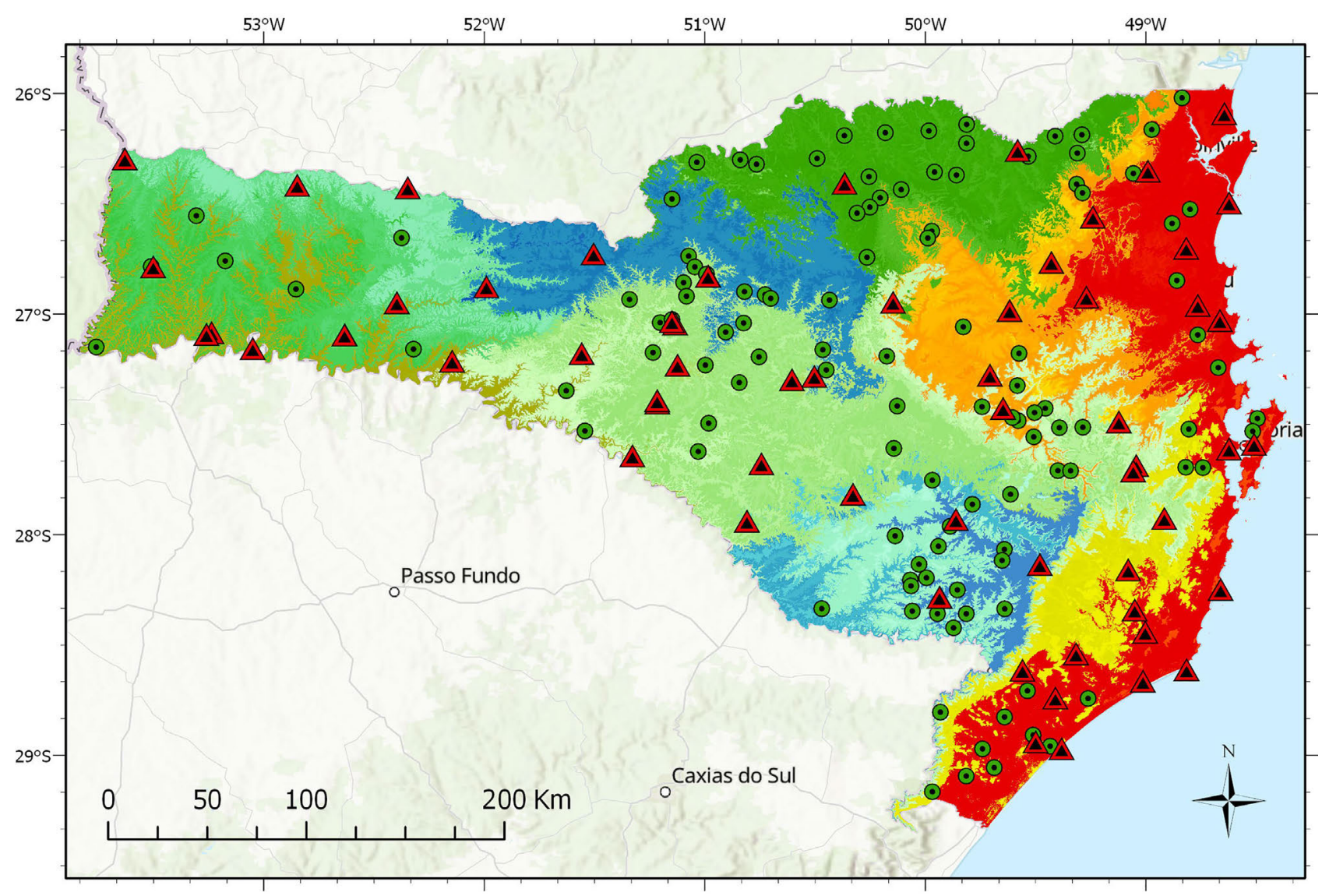

\begin{tabular}{|c|c|c|c|c|}
\hline Estações ativas (ago/2020) & $2 \mathrm{~A} 2$ & $2 \mathrm{C5}$ & $3 \mathrm{~B} 6$ & 4A7 \\
\hline Agrometeorológica & $2 \mathrm{~A} 3$ & $2 \mathrm{C6}$ & $3 \mathrm{~B} 7$ & 4A8 \\
\hline Meteorológica & | $2 \mathrm{~A} 4$ & $2 \mathrm{CC}$ & 3B8 & 4A9 \\
\hline & $2 \mathrm{~A} 5$ & $2 \mathrm{C} 8$ & 3B9 & $4 \mathrm{~B} 10$ \\
\hline Sub-reg. fisiográficas homogêneas & $2 \mathrm{~A} 6$ & $2 \mathrm{C} 9$ & $3 \mathrm{C} 10$ & $4 \mathrm{~B} 11$ \\
\hline IA1 & $2 \mathrm{~A} 7$ & 3A1 & $3 \mathrm{C} 11$ & $4 \mathrm{~B} 3$ \\
\hline $1 \mathrm{~A} 11$ & $2 \mathrm{~A} 8$ & 3A10 & 3C2 & 1 4B6 \\
\hline $1 \mathrm{~A} 2$ & $2 \mathrm{~A} 9$ & 3A11 & $3 \mathrm{C} 3$ & 4B7 \\
\hline $1 \mathrm{~A} 3$ & 2B1 & $3 \mathrm{~A} 2$ & 3C4 & 488 \\
\hline $1 \mathrm{~A} 4$ & $2 \mathrm{~B} 11$ & $3 A 3$ & $3 \mathrm{C5}$ & 4B9 \\
\hline IAS & $2 \mathrm{~B} 2$ & $3 A 4$ & | 3C6 & 510 \\
\hline $1 \mathrm{~A} 6$ & 2B3 & 3A5 & | $3 C 7$ & 511 \\
\hline 1A7 & $2 \mathrm{~B} 4$ & $3 A 6$ & $3 \mathrm{C} 8$ & 53 \\
\hline 1 A 9 & $2 \mathrm{~B} 5$ & $3 \mathrm{~A} 7$ & 3C9 & 54 \\
\hline $1 \mathrm{~B} 1$ & | $2 \mathrm{~B} 6$ & $3 A 8$ & 4A10 & 55 \\
\hline $1 \mathrm{~B} 2$ & | $2 \mathrm{~B} 7$ & 3А9 & 4A11 & 56 \\
\hline $1 \mathrm{~B} 3$ & $2 \mathrm{~B} 8$ & 3B10 & $4 \mathrm{~A} 2$ & 57 \\
\hline $1 \mathrm{~B} 4$ & $2 \mathrm{~B} 9$ & 3B11 & $4 A 3$ & 58 \\
\hline $2 \mathrm{~A} 1$ & $2 \mathrm{C} 2$ & 3B3 & $4 \mathrm{~A} 4$ & 59 \\
\hline $2 \mathrm{~A} 10$ & $2 \mathrm{C} 3$ & 3B4 & 4 AA5 & \\
\hline $2 \mathrm{~A} 11$ & $2 \mathrm{C} 4$ & $3 \mathrm{~B} 5$ & 4A6 & \\
\hline
\end{tabular}

Figura 4 - Mapa da distribuição espacial das estações de observação climática ativas em agosto de 2020 em Santa Catarina sobre as sub-regiões fisiográficas homogêneas.

representada e o acesso entre a localização atual e a localização proposta. As estações ativas que não coincidem com um ponto de estação proposta, mas estão distantes até $25 \mathrm{~km}$ de um ponto proposto, devem ser realocadas até o ponto proposto mais próximo (Fig. 6A). As estações ativas que não coincidem com um ponto proposto e que estão distantes mais de $25 \mathrm{~km}$ do ponto proposto mais próximo, devem ser utilizadas para substituir qualquer um dos pontos propostos restantes. Caso se opte por manter as estações ativas nos seus locais de origem, serão necessárias 145 novas estações para cobrir $100 \%$ das sub-regiões fisiográficas.

A nova proposta de distribuição espacial das estações de observação climática para o estado de Santa Cata- 


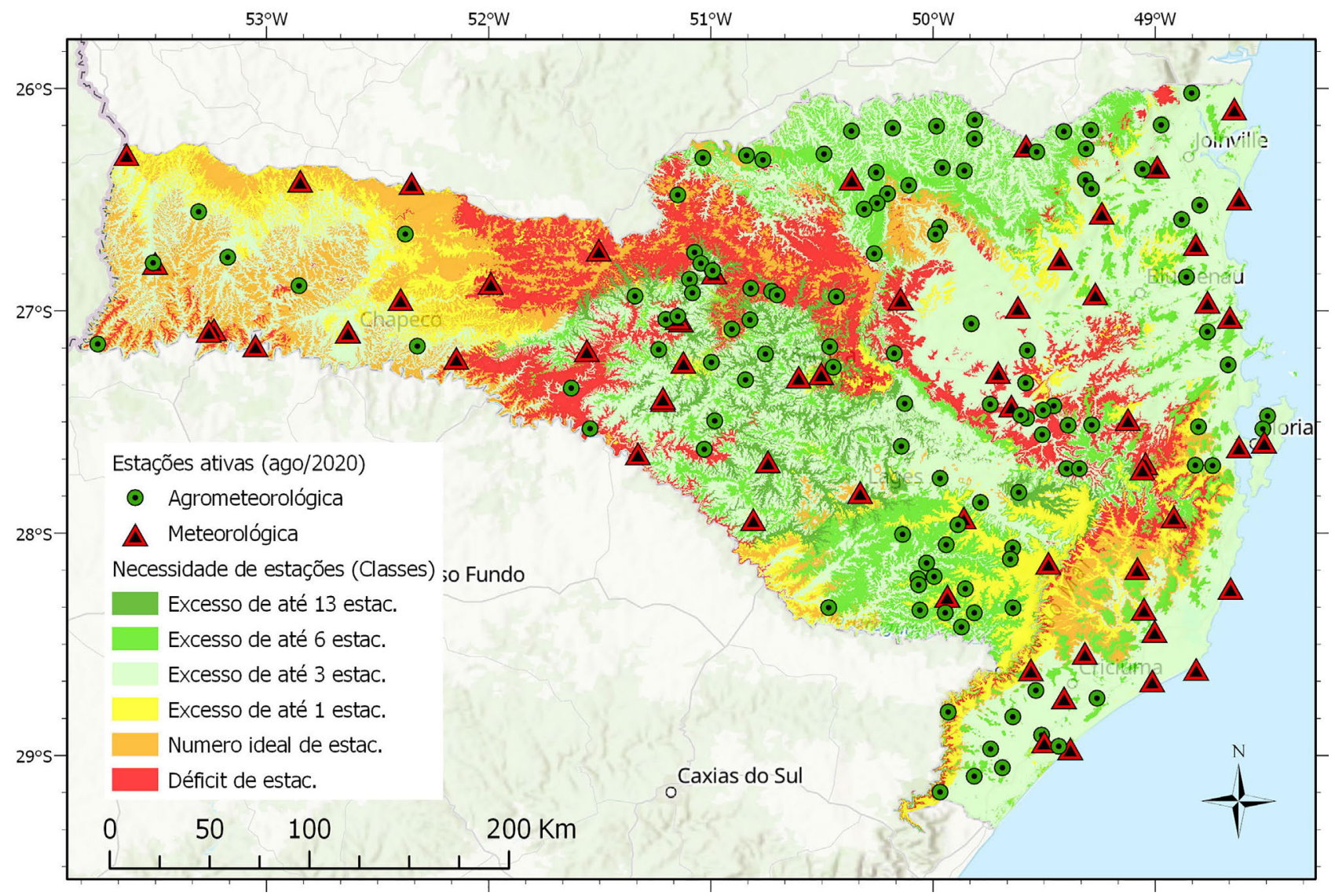

Figura 5 - Mapa da distribuição espacial das estações de observação climática ativas em agosto de 2020 em Santa Catarina sobre as sub-regiões fisiográficas homogêneas. As sub-regiões foram classificadas de acordo com o excedente ou déficit de estações, de acordo com as recomendações da OMM.

Tabela 2 - Quantidade de estações de observação climática existentes em agosto de 2010, quantidade de estações de observação climática necessárias e diferença entre a quantidade de estações de observação climática necessárias e a quantidade de estações de observação climática existentes por sub-região fisiográfica em Santa Catarina.

\begin{tabular}{lcccc}
\hline $\begin{array}{l}\text { Sub-região } \\
\text { fisiográfica }\end{array}$ & $\begin{array}{c}\text { Área } \\
\left(\mathrm{km}^{2}\right)\end{array}$ & $\begin{array}{c}\text { Estações exis- } \\
\text { tentes - Ee }(08 / \\
2020)\end{array}$ & $\begin{array}{c}\text { Estações } \\
\text { necessárias - } \\
\text { En }\end{array}$ & $\begin{array}{c}\text { Diferença } \\
(\text { Ee - En })\end{array}$ \\
\hline 1A1 & 11482 & 9 & 6 & 3 \\
1A11 & 4 & 0 & 1 & -1 \\
1A2 & 1532 & 8 & 1 & 7 \\
1A3 & 131 & 3 & 1 & 2 \\
1A4 & 207 & 3 & 1 & 2 \\
1A5 & 2 & 0 & 1 & -1 \\
1A6 & 1 & 0 & 1 & -1 \\
1A7 & 2 & 0 & 1 & -1 \\
1A9 & 0 & 0 & 1 & -1 \\
1B1 & 6 & 2 & 1 & 1 \\
1B2 & 300 & 6 & 1 & 5 \\
1B3 & 1 & 0 & 1 & -1 \\
1B4 & 4 & 0 & 1 & -1 \\
2A1 & 0 & 0 & 1 & -1 \\
2A10 & 1 & 0 & & 1 \\
\hline
\end{tabular}

\begin{tabular}{lcccc} 
continua & \multicolumn{5}{c}{} \\
\hline $\begin{array}{l}\text { Sub-região } \\
\text { fisiográfica }\end{array}$ & $\begin{array}{c}\text { Área } \\
\left(\mathrm{km}^{2}\right)\end{array}$ & $\begin{array}{c}\text { Estações exis- } \\
\text { tentes - Ee }(08 / \\
2020)\end{array}$ & $\begin{array}{c}\text { Estações } \\
\text { necessárias - } \\
\text { En }\end{array}$ & $\begin{array}{c}\text { Diferença } \\
(\text { Ee - En) }\end{array}$ \\
\hline 2A11 & 93 & 0 & 1 & -1 \\
2A2 & 95 & 0 & 1 & -1 \\
2A3 & 2691 & 5 & 1 & 4 \\
2A4 & 2729 & 4 & 1 & 3 \\
2A5 & 1726 & 4 & 1 & 3 \\
2A6 & 698 & 2 & 1 & 1 \\
2A7 & 543 & 4 & 1 & 3 \\
2A8 & 67 & 0 & 1 & -1 \\
2A9 & 122 & 2 & 1 & 1 \\
2B1 & 32 & 2 & 1 & 1 \\
2B11 & 1 & 0 & 1 & -1 \\
2B2 & 1387 & 9 & 1 & 8 \\
2B3 & 1792 & 2 & 1 & 1 \\
2B4 & 1764 & 1 & 1 & 0 \\
2B5 & 34 & 0 & 1 & -1 \\
\hline & & & 1 & 3 \\
\hline
\end{tabular}


Tabela 2 - continua

\begin{tabular}{|c|c|c|c|c|}
\hline $\begin{array}{l}\text { Sub-região } \\
\text { fisiográfica }\end{array}$ & $\begin{array}{l}\text { Área } \\
\left(\mathrm{km}^{2}\right)\end{array}$ & $\begin{array}{c}\text { Estações exis- } \\
\text { tentes - Ee (08/ } \\
2020)\end{array}$ & $\begin{array}{c}\text { Estações } \\
\text { necessárias - } \\
\text { En } \\
\end{array}$ & $\begin{array}{l}\text { Diferença } \\
\text { (Ee - En) }\end{array}$ \\
\hline $2 \mathrm{~B} 6$ & 4 & 0 & 1 & -1 \\
\hline 2B7 & 13 & 0 & 1 & -1 \\
\hline 2B8 & 0 & 0 & 1 & -1 \\
\hline $2 \mathrm{~B} 9$ & 1 & 0 & 1 & -1 \\
\hline $2 \mathrm{C} 2$ & 416 & 0 & 1 & -1 \\
\hline $2 \mathrm{C} 3$ & 2230 & 4 & 1 & 3 \\
\hline $2 \mathrm{C} 4$ & 721 & 1 & 1 & 0 \\
\hline $2 \mathrm{C} 5$ & 117 & 1 & 1 & 0 \\
\hline $2 \mathrm{C} 6$ & 28 & 1 & 1 & 0 \\
\hline $2 \mathrm{C} 7$ & 29 & 2 & 1 & 1 \\
\hline $2 \mathrm{C} 8$ & 0 & 0 & 1 & -1 \\
\hline $2 \mathrm{C} 9$ & 1 & 0 & 1 & -1 \\
\hline 3A1 & 1 & 0 & 1 & -1 \\
\hline $3 \mathrm{~A} 10$ & 310 & 3 & 1 & 2 \\
\hline 3A11 & 338 & 3 & 1 & 2 \\
\hline $3 \mathrm{~A} 2$ & 2 & 0 & 1 & -1 \\
\hline $3 \mathrm{~A} 3$ & 17 & 0 & 1 & -1 \\
\hline $3 \mathrm{~A} 4$ & 9 & 0 & 1 & -1 \\
\hline $3 \mathrm{~A} 5$ & 2763 & 0 & 1 & -1 \\
\hline $3 \mathrm{~A} 6$ & 3547 & 1 & 2 & -1 \\
\hline $3 \mathrm{~A} 7$ & 3878 & 10 & 2 & 8 \\
\hline $3 \mathrm{~A} 8$ & 4362 & 15 & 2 & 13 \\
\hline 3A9 & 8187 & 9 & 4 & 5 \\
\hline 3B10 & 41 & 0 & 1 & -1 \\
\hline 3B11 & 68 & 0 & 1 & -1 \\
\hline 3B3 & 9 & 0 & 1 & -1 \\
\hline 3B4 & 15 & 0 & 1 & -1 \\
\hline 3B5 & 91 & 0 & 1 & -1 \\
\hline 3B6 & 836 & 1 & 1 & 0 \\
\hline 3B7 & 2244 & 5 & 1 & 4 \\
\hline 3B8 & 2891 & 6 & 1 & 5 \\
\hline 3B9 & 4778 & 12 & 2 & 10 \\
\hline $3 \mathrm{C} 10$ & 8 & 0 & 1 & -1 \\
\hline $3 \mathrm{C} 11$ & 12 & 0 & 1 & -1 \\
\hline
\end{tabular}

rina diferencia-se da rede ativa atual (Fig. 3) por considerar, além da dimensão espacial do território do Estado, a variabilidade estatística do relevo. Com essa nova proposta, as estações ficariam distribuídas entre as variáveis fisiográficas (altitude, índice de posição topográfica e desvio padrão da altitude) e entre as regiões fisiográficas, proporcionalmente em relação às áreas de suas classes (Fig. 7).

Para exemplificar, a região do estado acima de $1200 \mathrm{~m}$ de altitude é representada por uma pequena área

\begin{tabular}{|c|c|c|c|c|}
\hline $\begin{array}{l}\text { Sub-região } \\
\text { fisiográfica }\end{array}$ & $\begin{array}{c}\text { Área } \\
\left(\mathrm{km}^{2}\right)\end{array}$ & $\begin{array}{c}\text { Estações exis- } \\
\text { tentes - Ee }(08 / \\
2020)\end{array}$ & $\begin{array}{c}\text { Estações } \\
\text { necessárias - } \\
\text { En }\end{array}$ & $\begin{array}{c}\text { Diferença } \\
(\mathrm{Ee}-\mathrm{En})\end{array}$ \\
\hline $3 \mathrm{C} 2$ & 2 & 0 & 1 & -1 \\
\hline $3 \mathrm{C} 3$ & 28 & 0 & 1 & -1 \\
\hline $3 \mathrm{C} 4$ & 3197 & 2 & 2 & 0 \\
\hline $3 \mathrm{C} 5$ & 3324 & 5 & 2 & 3 \\
\hline $3 \mathrm{C} 6$ & 2447 & 2 & 1 & 1 \\
\hline $3 \mathrm{C} 7$ & 1296 & 2 & 1 & 1 \\
\hline $3 \mathrm{C} 8$ & 802 & 1 & 1 & 0 \\
\hline 3C9 & 2174 & 1 & 1 & 0 \\
\hline $4 \mathrm{~A} 10$ & 3661 & 8 & 2 & 6 \\
\hline $4 \mathrm{~A} 11$ & 491 & 4 & 1 & 3 \\
\hline $4 \mathrm{~A} 2$ & 0 & 1 & 1 & 0 \\
\hline $4 \mathrm{~A} 3$ & 12 & 0 & 1 & -1 \\
\hline $4 \mathrm{~A} 4$ & 2 & 0 & 1 & -1 \\
\hline $4 \mathrm{~A} 5$ & 1 & 1 & 1 & 0 \\
\hline $4 \mathrm{~A} 6$ & 15 & 0 & 1 & -1 \\
\hline $4 \mathrm{~A} 7$ & 603 & 1 & 1 & 0 \\
\hline $4 \mathrm{~A} 8$ & 1389 & 4 & 1 & 3 \\
\hline 4A9 & 650 & 3 & 1 & 2 \\
\hline $4 \mathrm{~B} 10$ & 5851 & 2 & 3 & -1 \\
\hline 4B11 & 1525 & 1 & 1 & 0 \\
\hline 4B3 & 1 & 0 & 1 & -1 \\
\hline $4 \mathrm{~B} 6$ & 0 & 0 & 1 & -1 \\
\hline 4B7 & 77 & 0 & 1 & -1 \\
\hline 4B8 & 75 & 2 & 1 & 1 \\
\hline 4B9 & 30 & 0 & 1 & -1 \\
\hline 510 & 4 & 4 & 1 & 3 \\
\hline 511 & 2029 & 3 & 1 & 2 \\
\hline 53 & 2 & 0 & 1 & -1 \\
\hline 54 & 0 & 0 & 1 & -1 \\
\hline 55 & 1 & 0 & 1 & -1 \\
\hline 56 & 0 & 0 & 1 & -1 \\
\hline 57 & 3 & 0 & 1 & -1 \\
\hline 58 & 3 & 0 & 1 & -1 \\
\hline 59 & 3 & 0 & 1 & -1 \\
\hline
\end{tabular}

(Fig. 3A), que hoje possui 18 estações operando. Na nova proposta, essa mesma área poderia ser representada por 8 estações (Fig. 7) bem distribuídas entre as sub-classes fisiográficas que a compõem.

\section{Conclusão}

A rede de estações de observação climática de Santa Catarina é formada por uma quantidade de estações suficiente para representar climaticamente o território, porém 


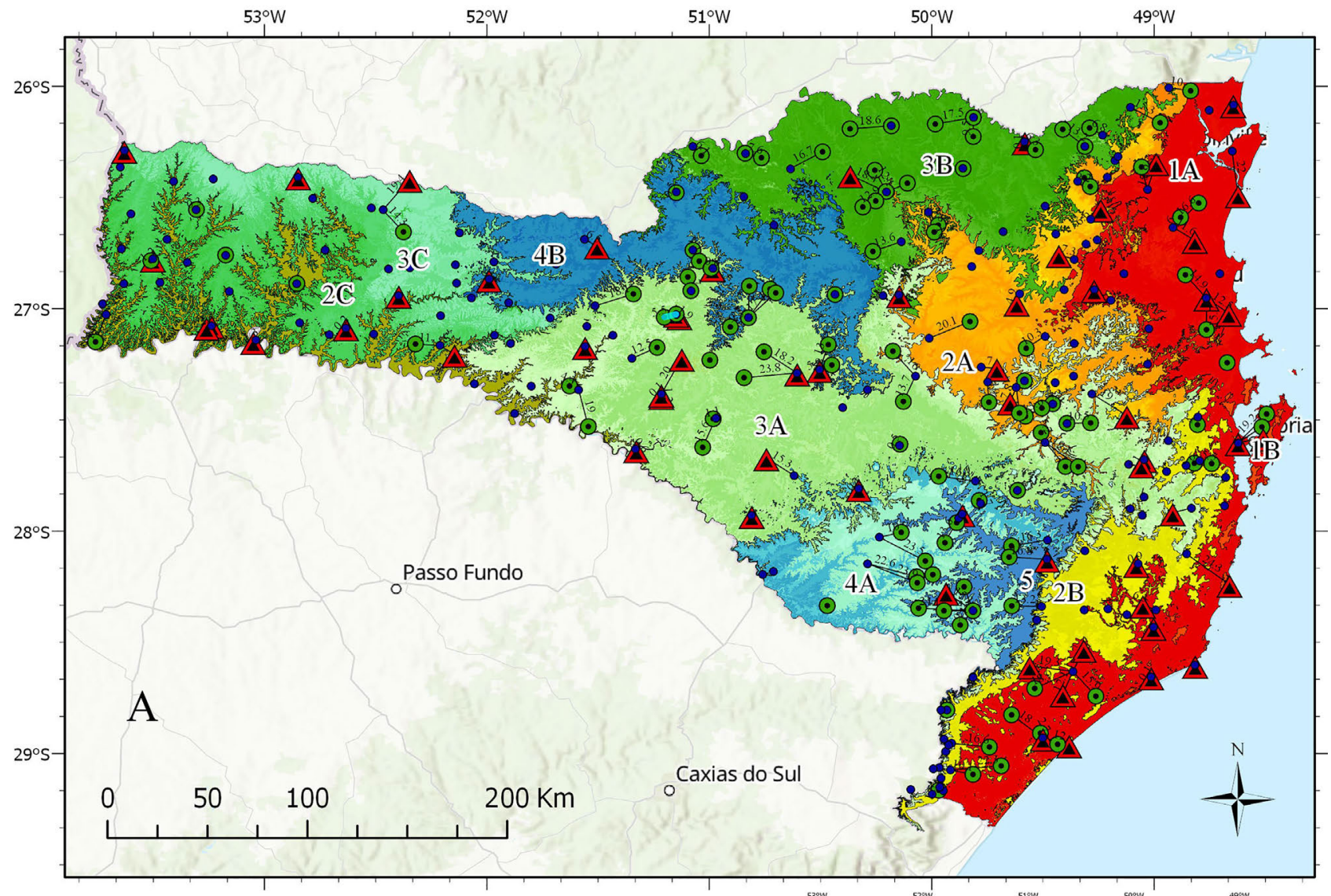

\begin{tabular}{l} 
Estações ativas (ago/2020) \\
Agrometeorológica \\
Peteorológica \\
Proposta para rede de estações \\
\hline Lim. reção mais próxima (km) \\
Sub-reg. fisiográficas homogêneas \\
$1 \mathrm{~A} 1$ \\
$1 \mathrm{~A} 11$ \\
$1 \mathrm{~A} 2$ \\
$1 \mathrm{~A} 3$ \\
$1 \mathrm{~A} 4$ \\
$1 \mathrm{~A} 5$ \\
$1 \mathrm{~A} 6$ \\
$1 \mathrm{~A} 7$ \\
$1 \mathrm{~A} 9$ \\
$1 \mathrm{~B} 1$ \\
$1 \mathrm{~B} 2$ \\
$1 \mathrm{~B} 3$ \\
$1 \mathrm{~B} 4$
\end{tabular}

\begin{tabular}{|l|l|l|l|}
\hline $2 A 1$ & $2 C 3$ & $3 B 5$ & $4 A 7$ \\
\hline $2 A 10$ & $2 C 4$ & $3 B 6$ & $4 A 8$ \\
\hline $2 A 11$ & $2 C 5$ & $3 B 7$ & $4 A 9$ \\
\hline $2 A 2$ & $2 C 6$ & $3 B 8$ & $4 B 10$ \\
\hline $2 A 3$ & $2 C 7$ & $3 B 9$ & $4 B 11$ \\
\hline $2 A 4$ & $2 C 8$ & $3 C 10$ & $4 B 3$ \\
\hline $2 A 5$ & $2 C 9$ & $3 C 11$ & $4 B 6$ \\
\hline $2 A 6$ & $3 A 1$ & $3 C 2$ & $4 B 7$ \\
\hline $2 A 7$ & $3 A 10$ & $3 C 3$ & $4 B 8$ \\
\hline $2 A 8$ & $3 A 11$ & $3 C 4$ & $4 B 9$ \\
\hline $2 A 9$ & $3 A 2$ & $3 C 5$ & 510 \\
\hline $2 B 1$ & $3 A 3$ & $3 C 6$ & 511 \\
\hline $2 B 11$ & $3 A 4$ & $3 C 7$ & 53 \\
\hline $2 B 2$ & $3 A 5$ & $3 C 8$ & 54 \\
\hline $2 B 3$ & $3 A 6$ & $3 C 9$ & 55 \\
\hline $2 B 4$ & $3 A 7$ & $4 A 10$ & 56 \\
\hline $2 B 5$ & $3 A 8$ & $4 A 11$ & 57 \\
\hline $2 B 6$ & $3 A 9$ & $4 A 2$ & 58 \\
\hline $2 B 7$ & $3 B 10$ & $4 A 3$ & 59 \\
\hline $2 B 8$ & $3 B 11$ & $4 A 4$ & \\
\hline $2 B 9$ & $3 B 3$ & $4 A 5$ & \\
\hline $2 C 2$ & $3 B 4$ & $4 A 6$ & \\
\hline
\end{tabular}

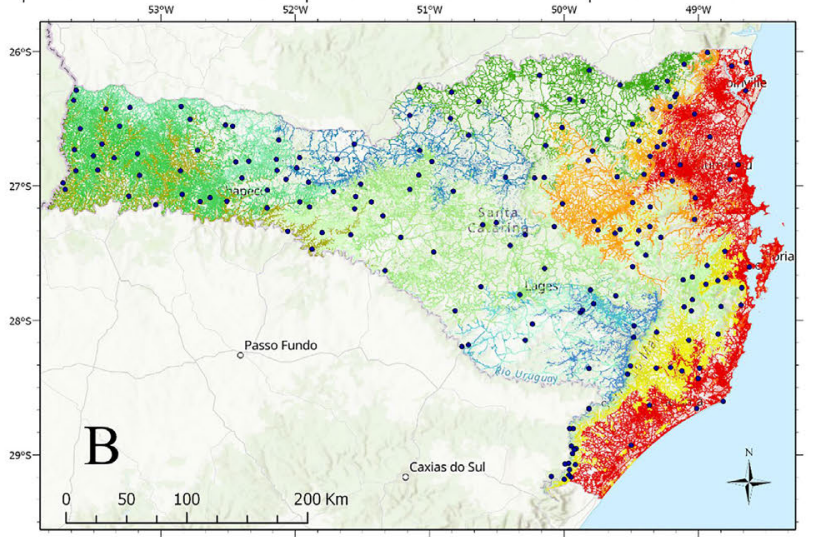

Figura 6 - (A) Proposta de realocação espacial das estações de observação climática para cobrir 100\% das regiões e sub-regiões fisiográficas de Santa Catarina de acordo com as recomendações da OMM. (B) Proposta de distribuição espacial das estações de observação climática nas áreas das sub-regiões fisiográficas com acesso viário e cobertura de telefonia móvel.

a atual distribuição espacial não representa a variabilidade fisiográfica do estado, segundo os critérios mínimos da OMM. As estações atualmente ativas, principalmente as agrometeorológicas, tiveram seus sítios escolhidos com base em objetivos relacionados majoritariamente à agri- cultura, por isso observa-se concentrações de estações em regiões fisiograficamente homogêneas. Para atender às recomendações da $\mathrm{OMM}$ em relação à representatividade climática, o estado de Santa Catarina precisa otimizar a distribuição espacial das estações de observação existentes 

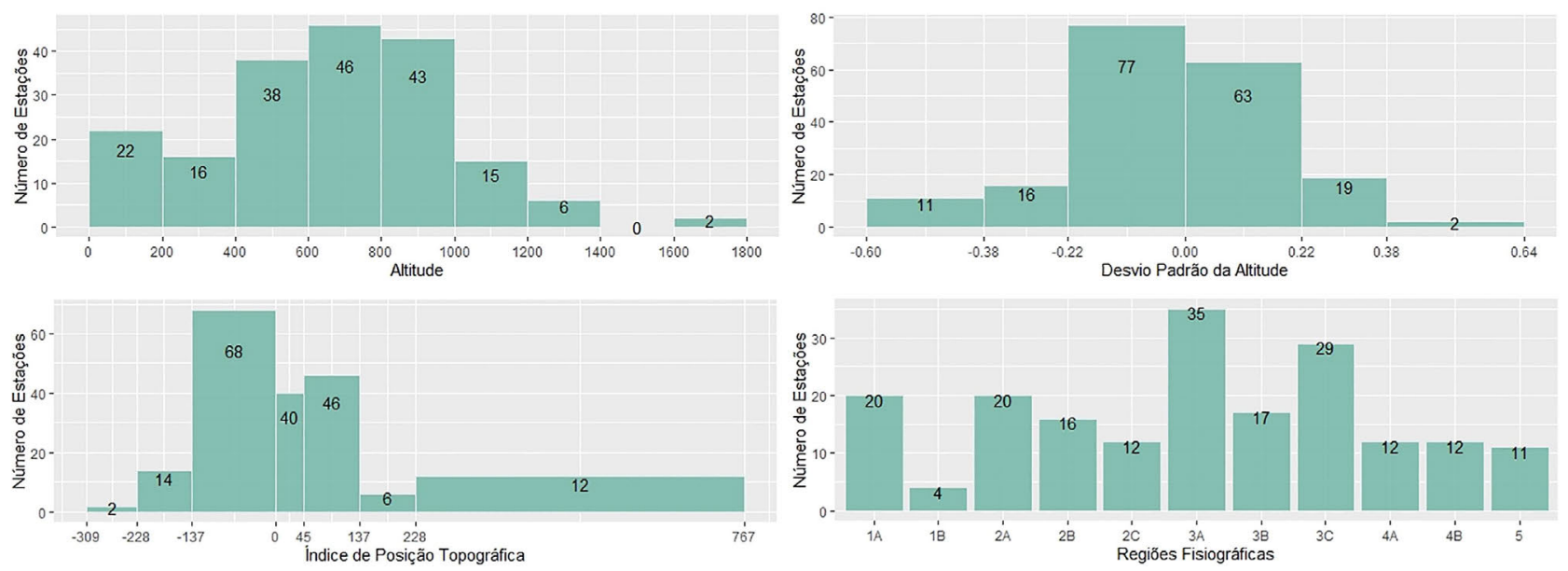

Figura 7 - Número de estações de observação climática propostas por classe de altitude, desvio padrão da altitude, índice de posição topográfica (TPI) e regiões fisiográficas homogêneas.

em seu território. Esse processo poderá ser feito através do remanejamento de estações das sub-regiões fisiográficas onde haja excedente para as sub-regiões fisiográficas com déficit. A proposta de distribuição espacial das estações de observação climática aqui apresentada, pode servir de base para uma adequação otimizada, pois considera as estações existentes, a variabilidade fisiográfica e a capacidade mínima logística para um remanejamento. Mas para remanejar as estações e melhorar a representatividade climática da rede é preciso, ainda, avaliar o histórico de dados de cada estação de observação climática (Vianna et al., 2018). Também é importante definir o sítio de instalação segundo as orientações da $\mathrm{OMM}$ e estar atento à acurácia dos metadados espaciais e temporais (Vianna e Massignan, 2018).

\section{Referências}

AMATULLI, G.; DOMISCH, S.; TUANMU, M.N.; PARMENTIER, B.; RANIPETA, A.; MALCZYK, J.; JETZ, W. Data Descriptor: A suite of global, cross-scale topographic variables for environmental and biodiversity modeling. Sci Data. v. 5, 180040, 2018.

BLAINSKI, E.; GARBOSSA, L.H.P.; ANTUNES, E.N. Estações Hidrometeorológicas Automáticas: Recomendações Técnicas para Instalação. Florianópolis: Epagri, 2012.

BRAGA, H.J.; GHELLERE, R. Proposta de diferenciação climática para o Estado de Santa Catarina. In: 11 Congresso Brasileiro de Agrometeorologia., 1999, Florianópolis, SC. Anais. Florianópolis: Epagri, 1999. 1 CD-ROM.

BRAGA, H.J.; RICCE, W. DO S.; PANDOLFO, C.; GARBOSA, L.H.P.; MASSIGNAM, A.M.; BLAINSKI, E.; VIEIRA, H.J. Agrometeorologia Catarinense: Estações Convencionais. Florianópolis, Epagri, 2015.

ESRI. ArcGis Pro. Point Density (Spatial Analyst). Tool Reference. Disponível em: https://pro.arcgis.com/en/pro- app/tool-reference/spatial-analyst/point-density.htm. Acesso em: 06/08/2020. 2020.

ESRI. ArcGis Pro. How Principal Components Works. Tool Reference. Disponível em: https://pro.arcgis.com/en/proapp/tool-reference/spatial-analyst/how-principal-compo nents-works.htm. Acesso em: 06/08/2020. 2020a.

ESRI. ArcGis Pro. Iso Cluster Unsupervised Classification. Tool Reference. Disponível em: https://pro.arcgis.com/en/ pro-app/tool-reference/spatial-analyst/iso-cluster-unsuper vised-classification.htm. Acesso em: 06/08/2020. $2020 \mathrm{~b}$.

ESRI. ArcGis Pro. Viweshade Function. Tool Reference. Disponível em: https://pro.arcgis.com/en/pro-app/help/data/ imagery/viewshed-global-function.htm. Acesso em: 06/08/ 2020. 2020c.

ESRI. ArcGis Pro. Euclidean Distance Function. Tool Reference. Disponível em: https://pro.arcgis.com/en/pro-app/ tool-reference/spatial-analyst/euclidean-distance.htm. Acesso em: 06/08/2020. 2020d.

ESRI. ArcGis Pro. Euclidean Distance Function. Tool Reference. Disponível em: https://pro.arcgis.com/en/pro-app/ 2.6/tool-reference/data-management/how-create-randompoints-works.htm. Acesso em: 18/01/2021. 2020e.

JENNESS, J.; REA, S.U.A.; TIN, F. Some Thoughts on Analyzing Topographic Habitat Characteristics Surface Area. Flagstaff: Janness Enterprises, 26 p., 2007.

KASSAMBARA, A. Practical Guide to Cluster Analysis in R: Unsupervised Machine Learning. 1st ed. Marseille: STHDA, 2017.

NASA/METI/AIST/ASTER Science Team. ASTER DEM Product. Distribuído por: NASA EOSDIS Land Processes DAAC. Disponível em: https://doi.org/10.5067/ASTER/ AST14DEM.003. Acesso em: 04/08/2020. 2001

SANTA CATARINA. Atlas Geográfico de Santa Catarina: Diversidade da Natureza - Fascículo 2. Isa de Oliveira Rocha (org.). Florianópolis: Ed. UDESC, 188 p., 2014.

TAGIL, S.; JENNESS, J. GIS-Based Automated Landform Classification and Topographic, Landcover and Geologic Attributes of Landforms Around the Yazoren Polje, Turkey. Journal of Applied Sciences, v. 8, n. 6, p. 910-921, 2008. 
VIANNA, L.F.; PERIN, E.B.; RICCE, W. DA S.; MASSIGNAM, A.M.; PANDOLFO, C. Bancos de dados meteorológicos: análise dos metadados das estações de observação climática no Estado de Santa Catarina, Brasil. Rev. Bras. Meteorol., v. 32, n. 1, p. 53-64, 2017.

VIANNA, L.F.; MASSIGNAM, A.M. Distribuição espacial dos metadados temporais e qualidade dos metadados espaciais das estações hidrometeorológicas do Estado de Santa Catarina para climatologia. Rev. Bras. Meteorol., v. 33, n. 3, p. 412-425, 2018.

WMO - World Meteorological Organization. Guide to the Global Observing System, WMO $n^{\circ} .488,3^{a}$ ed. Genebra: WMO, 2007.
WMO - World Meteorological Organization. Guide to Meteorological Instruments and Methods of Observation: (CIMO Guide). Geneva: WMO, 2014.

\section{Internet resources}

Associação Brasileira de Telecomunicações, http://www.teleco care.com.br/telebrasil/mapa_erb.php.

Geofrabrik, https://download.geofabrik.de/south-america/brazil. html.

License information: This is an open-access article distributed under the terms of the Creative Commons Attribution License (type CC-BY), which permits unrestricted use, distribution and reproduction in any medium, provided the original article is properly cited. 